\title{
Long-Term Outcomes After Open Repair for Ruptured Abdominal Aortic Aneurysm
}

\author{
Andreas Reite $^{5}$ (1) $\cdot$ Kjetil Søreide $^{2,3} \cdot$ Jan Terje Kvaløy $^{4,5} \cdot$ Morten Vetrhus $^{1,6}$
}

Published online: 9 March 2020

(C) The Author(s) 2020

\begin{abstract}
Background Early mortality in ruptured abdominal aneurysm (rAAA) is high, but data on long-term outcome are scarce. The aim of this study was to investigate the long-term outcome in survivors after open surgery for rAAA in well-defined population.

Methods This is a population-based, observational long-term follow-up (beyond 30-day mortality) study of patients surgically treated for rAAA from 2000 through 2014. Long-term survival was analysed using Kaplan-Meier estimates and compared to the general population by analyses of relative survival.

Results Out of 178 patients operated for rAAA, 95 patients (55\%) either died in the perioperative period, were referred from other hospitals or were lost to follow-up (two patients). Altogether 83 patients were eligible for longterm outcomes: 72 men and 11 women. Estimated median crude survival time was 6.5 years [95\% confidence interval (CI) 4.8-8.2]. Men had a median survival of 7.3 years (95\% CI 5.1-9.4) versus 5.4 years in females (95\% CI 3.5-7.3) $(P=0.082)$. Reinterventions during follow-up occurred in $31(37 \%)$. Relative survival demonstrated a slightly higher risk of death in the rAAA population compared to the general age- and gender-matched population. Age, but not comorbidities, had a significant influence on long-term survival.

Conclusion For survivors beyond 30 days after surgery for rAAA, long-term survival compares well to that of an age- and sex-matched population. A high frequency of cardiovascular comorbidities did not seem to affect long-term survival.
\end{abstract}

Andreas Reite

reanen@sus.no

1 Department of Surgery, Vascular Surgery Unit, Stavanger University Hospital, PO Box 8100, 4068 Stavanger, Norway

2 Department of Gastrointestinal Surgery, Stavanger University Hospital, Stavanger, Norway

3 Department of Clinical Medicine, University of Bergen, Bergen, Norway

4 Research Department, Stavanger University Hospital, Stavanger, Norway

5 Department of Mathematics and Physics, University of Stavanger, Stavanger, Norway

6 Department of Clinical Science, University of Bergen, Bergen, Norway

\section{Introduction}

Surgery for ruptured abdominal aortic aneurysms (rAAA) is still challenging with perioperative mortality rates ranging from 40 to $60 \%$ in most studies [1,2]. Reports suggest a wide variation in perioperative mortality $[2,3]$. The incidence of rAAA and the perioperative mortality has declined over the last two decades, and lower mortality may be due to the implementation of endovascular repair and improved pre- and in-hospital care [2, 4, 5]. Previous studies have mainly focused on the perioperative period, and less attention has been paid to the long-term outcome in patients who do survive the first 30 days. The latest Cochrane review [6] stated that long-term data on survival 
and late complications are scarce after both endovascular and open treatments of rAAA. Factors that may affect survival past the perioperative period in patients operated for rAAA have been described in a few previous reports, and the results are not consistent [7, 8]. Few populationbased studies on long-term outcome with an adequate follow-up time are available [9]; the majority of these studies are from a pre-selected population, i.e. not population-based, and thus, the generalizability of the findings is questionable.

The aim of this study was to present data on long-term survival, including relative survival and factors related to long-term survival and excess mortality in a well-defined Norwegian population.

\section{Methods}

The study was approved by the Regional Committee for Medical and Health Research Ethics North (REK Nord 2011/918).

\section{Study population}

Stavanger University Hospital (SUH) has a primary catchment population of 360,000 and is the only hospital serving the population of South Rogaland County. SUH also receives tertiary referrals from other hospitals, covering a total population of about 500,000 inhabitants. The epidemiology and perioperative mortality for rAAA in this region have been reported earlier $[1,10]$.

\section{Study design and inclusion criteria}

The study is a single-centre population-based retrospective observational study, including all patients from SUHs primary catchment area operated for rAAA during the period from January 2000 to December 2014. The study was confined to juxta-renal and infra-renal primary rAAA only. To avoid referral or survival bias from transferred patients, only patients from the primary catchment area were included.

Survival and mortality data were obtained from the prospectively maintained electronic patient record database at the hospital. The study has, to the best of our abilities, been conducted according to the STROBE guidelines for observational studies [11].

\section{Definitions}

rAAA was confirmed by extravasation of blood on computed tomography (CT) and/or a periaortic haematoma on operation. Secondary aneurysms (anastomotic aneurysms after the previous repair of aneurysm or atherosclerosis) and isolated iliac aneurysms without any aortic component were excluded.

Comorbidity was defined as evidence in past medical history of organ or system diseases. Heart disease included any heart condition including congestive heart failure, rhythm disturbances and previous ischaemic heart disease.

The patients' physical status was classified according to the American Society of Anaesthesiologists (ASA) Physical Status Classification [12] into five classes where class 1 is a normally healthy patient and class 5 is a moribund patient who is not expected to survive for $24 \mathrm{~h}$ with or without operation.

Complications after surgery were classified as minor or major, major defined as complications requiring reintervention under general anaesthesia or the presence of multiorgan failure.

The Oxford Vascular study [13] showed that two-thirds of acute abdominal aortic aneurysm (AAA) events occur at age $\geq 75$ years; we used this age as a cut-off to dichotomize patients into 'older' ( $\geq 75$ years) or 'younger' ( $<75$ years).

\section{Survival data}

Survival and mortality data were obtained from the electronic patient record database where vital status is regularly updated from the Norwegian national population data registry. Patients alive as per 6 October 2018 were censored.

\section{Statistical analyses}

All analyses were performed with the Statistical Package for Social Sciences (v 23; IBM, Armonk, NY, USA) and R 3.4.3 [14] The R package 'relsurv' version 2.0-9 was used for the relative survival calculations [15].

Mann-Whitney test was used to test for differences between continuous variables and Pearson's Chi-squared test for categorical values.

Relative survival analysis was calculated as the probability of patients operated for rAAA (excluding rAAA with 30-day mortality) surviving to a given time post-operatively divided by the probability that persons in the general population would survive to that time, given the same age, gender and year of birth distributions. Population survival was calculated with Norwegian population lifetime tables from Statistics Norway [16].

For crude survival calculations, Kaplan-Meier estimates were used and investigated by the log-rank test. For impact of covariates, first univariable Cox regression was performed. Then covariates with $P<0.2$ were included in a multivariable Cox regression analysis. 
All statistical tests were two-sided, and the level of significance was set at $P<0.050$.

\section{Results}

During the study period, 178 patients had an operation for rAAA (Fig. 1). All but one patient (endovascular repair) had an open operation.

Patients who died perioperatively (within 30 days) were excluded $(N=72)$ from the analyses. Patients referred from other institutions with rAAA $(N=21)$ were excluded for a correct epidemiological assessment. Finally, two patients had no follow-up data as they had left Norway. Thus, 83 patients were included in the study: $72(87 \%)$ males and $11(13 \%)$ females (Table 1). Altogether, 58 of 83 patients $(70 \%)$ died during follow-up.

\section{Patient characteristics}

Patient characteristics are given for females and men in Table 1. A substantial number of the patients had known heart disease: $31 \%(N=13)$ of the patients $<75$ years and $45 \%(N=17)$ of the elderly patients. Increasing age was associated with mortality (Table 2). No significant difference in comorbidity or rate of new interventions was found in a comparison between the different age groups.

\section{Complications, reinterventions and mortality}

Early ( $<30$ days) and late complications leading to surgical intervention are listed in Table 3: 24 early complications were registered in 18 patients (22\%) as four patients needed intervention for more than one complication.

Late complications were registered in five patients (6\%). One patient had a secondary rupture in the distal anastomosis that was successfully repaired, and two patients $(2 \%)$ were diagnosed with graft infection that presented with a psoas abscess adjacent to the aortic graft; one patient died from septicaemia, while the other was successfully treated with antibiotics.

The cumulative number of reinterventions ranged from 1 to 4 per patient. The total number of reinterventions per patient had a significant impact on long-term survival (log rank, $P=0.009$ ).

Most patients experienced a temporary decrease in renal function with a median creatinine before surgery of 113 $\mu \mathrm{mol} / \mathrm{L}$ (range 53-500) and $145 \mu \mathrm{mol} / \mathrm{L}$ (range 56-677) after surgery. Eleven (13\%) patients had more than a threefold raise in creatinine after surgery, equivalent to acute renal failure. Four (5\%) patients developed persistent renal failure requiring permanent renal replacement therapy. Altogether 51 (61\%) patients were discharged to their home, $24(29 \%)$ to a short time stay in a nursing home and eight $(10 \%)$ to another hospital. The long-term functional outcome is not known.

The cause of death was known in 38 patients who died in hospital (Table 4): $32(84 \%)$ men with a median age of 74 years and $6(16 \%)$ females with a median age of 79 years. Seven of the 83 patients $(8 \%)$ included in the study died of aneurysm-related causes. Four (11\%) patients died between 30 and 90 days after surgery from renal, cardiac and/or pulmonary complications of rAAA.

Cancer $(34 \%, N=13)$, cardiovascular (including stroke, $26 \%, N=10)$ and pulmonary diseases $(16 \%, N=6)$ were

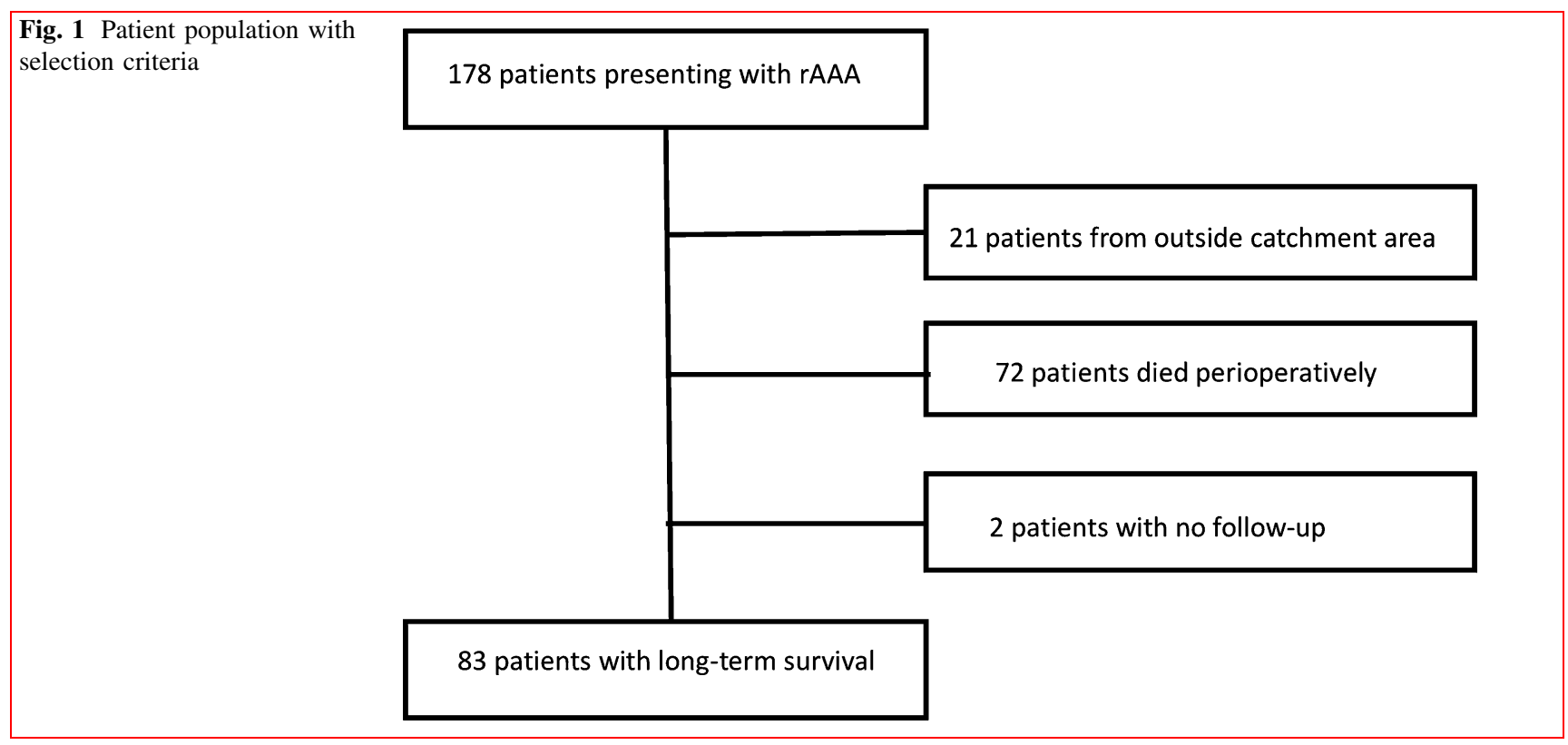


Table 1 Patient characteristics stratified by gender

\begin{tabular}{llll}
\hline Variables & Male $(N=72)$ & Female $(N=11)$ \\
\hline Age & $72.8(9.9)$ & $84.1(8.9)$ & $72(18.9)$ \\
Aneurysm size (mm) & $76.0(18.1)^{\mathrm{a}}$ & $5.4(3.1)$ & 0.021 \\
Survival (year) & $5.8(4.5)$ & $2(12.6)$ & 0.615 \\
Intensive care (days) & $5(15.6)$ & $0(5.6)$ & 0.184 \\
Ventilator (days) & $1(12.8)$ & $2400(3132)$ & 0.137 \\
Bleeding (mL) & $2400(2602)$ & $143(59.0)$ & 0.186 \\
Operation time (min) & $158(63.6)$ & $60(15.2)$ & 0.893 \\
Preoperative pulse rate & $67(16.4)$ & $80(11.4)$ & 0.379 \\
Preoperative systolic blood pressure (mm Hg) & $70(15.7)$ & $1750(839)$ & $7(64 \%)$ \\
Blood transfusion (mL) & $1750(1375)$ & $6(55 \%)$ & 0.294 \\
Heart disease & $23(32 \%)$ & 0 & 0.205 \\
Coronary disease & $18(25 \%)$ & $3(27 \%)$ \\
Pulmonary disease & $7(10 \%)$ & 0.877 \\
Cerebrovascular disease & $6(8 \%)$ & $1(9 \%)$ \\
Renal failure & $3(4 \%)$ & $2(18 \%)$ \\
Diabetes Mellitus & $6(8 \%)$ & $3(27 \%)$ \\
Beta-blocker & $10(14 \%)$ & $4(3-4)$ & 0.042 \\
Statins & $24(33 \%)$ & 0.044 \\
ASA & $4(2-5)^{\mathrm{b}}$ & 0.060 \\
\hline Da & & 0.490 \\
\end{tabular}

Data presented as counts $(\%)$ or median $( \pm \mathrm{SD})$. All values are median with range or percentage in parentheses. Missing data: a: six cases, b: three cases

Table 2 Risk factor analysis for death in rAAA (30-day mortality excluded)

\begin{tabular}{lll}
\hline Variables & Hazard ratio $(95 \% \mathrm{CI})$ & $P$ \\
\hline Age & $1.1(1.0-1.1)$ & 0.002 \\
Sex & $2.0(1.0-4.1)$ & 0.066 \\
Coronary heart disease & $0.9(0.5-1.5)$ & 0.604 \\
Renal failure & $2.7(0.4-19.5)$ & 0.327 \\
Pulmonary disease & $0.4(0.2-1.1)$ & 0.093 \\
Cerebrovascular disease & $0.5(0.2-1.2)$ & 0.121 \\
Diabetes mellitus & $0.9(0.3-2.4)$ & 0.866 \\
Transfusion (SAG) $>1200 \mathrm{~mL}$ & $0,9(0.5-1.5)$ & 0.608 \\
Statins & $0.9(0.5-1.6)$ & 0.747 \\
Beta-blocker & $2.7(0.8-8.5)$ & 0.101 \\
\hline
\end{tabular}

the three major causes of death in the 38 patients with a confirmed cause of death. The cause of death was unknown for all 20 patients who died outside of hospital.

\section{Crude, long-term and relative survival}

The longest follow-up was 17.6 years, and the median follow-up was 5.7 years $[95 \%$ confidence interval (CI)
4.6-7.0]. At the end of the study, 25 (30\%) patients were still alive. The estimated median crude survival (excluding 30-day mortality) (Fig. 2) was 6.5 years (95\% CI 4.8-8.2). For males, the median survival time was 7.3 years $(95 \% \mathrm{CI}$ 5.1-9.4), and for females, it was 5.4 (95\% CI 3.5-7.3). There was a non-significant difference in long-term survival between the genders $(P=0.082)$. Hazard ratio for relevant comorbidities is displayed in Table 2.

For patients $<75$ years the median survival was 9.2 years (95\% CI 3.6-14.9) and 5.4 years (95\% CI 3.4-7.4) for those $\geq 75$ years. The younger patients had a significantly longer survival (log rank, $P=0.003$ ) (Table 2).

In patients with known heart disease, median survival was 5.9 years (95\% CI 4.9-6.9), compared to 7.5 years (95\% CI 3.4-11.5) in those without. The difference in survival was not significant ( $\log$ rank, $P=0.195$ ). Patients with lung disease had a median survival 3.9 years $(95 \% \mathrm{CI}$ 0.2-7.6) compared to individuals with no lung disease 7.0 years (95\% CI 5.2-8.7), again the difference was not significant (log rank, $P=0.122$ ). One patient survived 30 days after being submitted with rAAA and ongoing CPR. This patient was still alive at the end of follow-up 14 years after surgery for rAAA.

The relative survival demonstrated only a slightly higher risk of death in the operated population the first 5 years 
Table 3 Early and late complications requiring surgical intervention, counts (\%)

\begin{tabular}{llc}
\hline Complications requiring intervention & $N=83$ & Mean time to intervention \\
\hline Early (<30 days) & & 11 days \\
Major amputation & $5(6 \%)$ & 7 days \\
Colectomy, partial or total & $5(6 \%)$ & 1 day \\
Open abdomen & $3(4 \%)$ & 8 days \\
Haemorrhage & $5(6 \%)$ & 3 days \\
Wound dehiscence & $3(4 \%)$ & 2 days \\
Revascularization lower limb & $3(4 \%)$ & 8 years \\
Late $(>30$ days) & & 1.5 years \\
Secondary rupture & $1(1 \%)$ & $2(2 \%)$ \\
Ventral hernia & $2(2 \%)$ & 0.5 year \\
Graft infection & & \\
\hline
\end{tabular}

Table 4 Cause of death in the 58 patients who died during follow-up (>30 days)

\begin{tabular}{lc}
\hline Cause of death & $N=58$ \\
\hline Cancer & 13 \\
Heart disease & 6 \\
Lung disease* & 6 \\
Cerebrovascular accident** & 4 \\
Dementia & 1 \\
Metachronous primary aneurysm & 2 \\
Post-operative complications, 30-90 days after surgery & 4 \\
Graft infection & 1 \\
Pancreatitis & 1 \\
Unknown & 20 \\
\hline
\end{tabular}

*Pneumonia or COPD-related, **stroke or intracranial haemorrhage

after surgery compared to an age- and sex-matched general population. As follow-up time approached 12 years, the estimated relative survival was lower in patients operated on for rAAA compared to the general population, but at the extreme of follow-up few patients were at risk and the findings are uncertain due to wide confidence intervals. The 12-year relative survival is displayed in Fig. 3.

\section{Discussion}

In this population-based observational study of patients undergoing open surgery for rAAA and surviving the first 30 days, actual survival was $64 \%$ after 5 years and 33\% after 10 years. For patients surviving the first 30 days after surgery for rAAA, long-term survival was good and comparable to that of an age- and sex-matched population. When excluding mortality related to the perioperative period, age was the only factor that significantly affected survival in patients with rAAA. The durability of repair was good, with a low aneurysm-related mortality. No data on long-term functional outcome were available, but a majority of patients were discharged to their home.

The prevalence of AAA and the incidence of rAAA have been reported to decline over the last two decades [17]. Survival from rAAA has increased in the same time period. This may have a multifactorial cause including improvements in pre-hospital care, better protocols and operative management and improved intensive care postoperatively [18].

The results from previously published studies on longterm survival after rAAA outside of Norway are not consistent $[8,9,19-28]$. Several investigators have concluded that long-term outcome for patients treated surgically for rAAA was worse than the population at large $[7,20]$. Vice versa, recent papers substantiate that long-term survival in rAAA is equal to or slightly lower than in the general population [8, 9, 21], and one study found that 10-16 months after surgery for rAAA the relative survival reached the same level as for an age- and gender-matched population [22].

The IMPROVE trial investigators have recently presented 3-year clinical outcome from the UK trial [29] with a three-year mortality after open repair of 54 versus $42 \%$ after endovascular repair. This is comparable to the crude survival after 3 years in our study representing a general, unselected cohort of all-comers to a single hospital (Fig. 2).

Age at time of presentation is a strong predictor of longterm survival [8]. Age was also found to be predictive of long-term survival in the current study. A study from the Swedish Vascular registry spanning 8 years found that in rAAA, both age and the proportion of patients with comorbidities increased over time, but interestingly no 


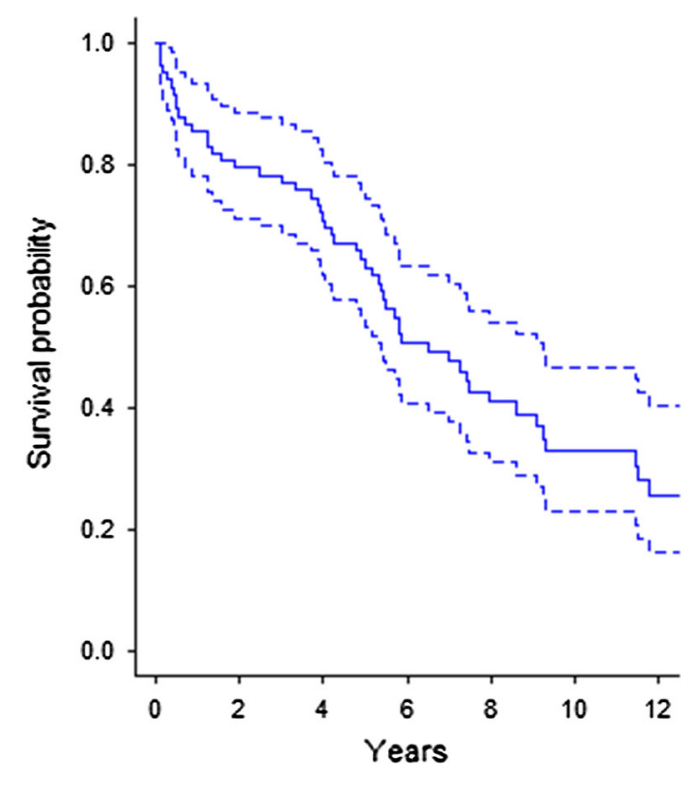

$\begin{array}{llllllll}\text { At risk: } & 83 & 66 & 58 & 35 & 23 & 16 & 10\end{array}$

Fig. 2 Crude survival. Legend: interval from time of operation to 12 years post-operatively. Number at risk displayed below. The solid line represents the crude survival, and the dotted lines represent $95 \% \mathrm{CI}$

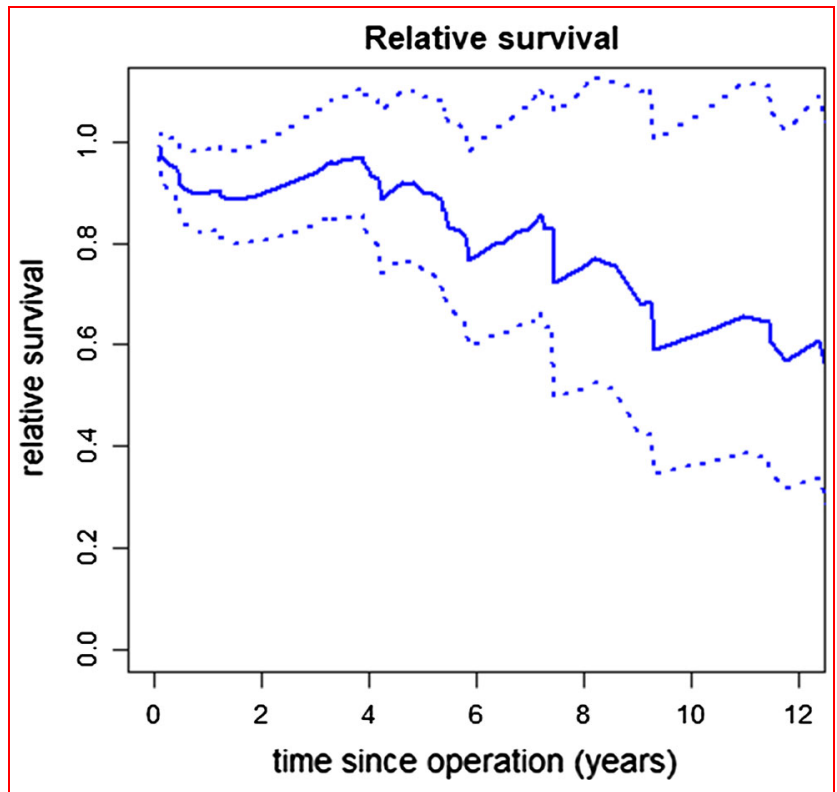

Fig. 3 Relative survival. Legend: interval from time of operation to 12 years post-operatively. The solid line represents the relative survival, and the dotted lines represent $95 \%$ CI

significant difference in long-term survival over time [21]. In a comparison of gender and long-time survival, we did not find any significant difference $(P=0.449)$. Most studies on long-term survival describe a significant difference in disfavour of women in survival $[7,8,21]$ : higher expansion rate, likelihood of rupture at a smaller diameter and in general higher mortality $[30,31]$.

Surgical reintervention was needed in 31 patients (37\%); the majority were confined to the first 30 days after primary surgery. The few late complications $(5 \%)$ were registered after a median follow-up of 5.7 years and are comparable to the results from a meta-analysis of three randomized controlled trials (IMPROVE, AJAX and ECAR) [32] which reported a compiled late reintervention rate of $2.2 \%$ in open repair after a short follow-up time of 1 year ( $>30$ days to 1 year). In the ECAR trial [33], haemodialysis beyond 30 days was reported to be $3.9 \%$ in the open group, similar to our findings.

In the present study, only one patient was treated for a paraanastomotic aneurysm (1\%), and it seems reasonable to state that this is an underestimate as patients have no imaging scheduled later than 1 year after surgery. An earlier study reported the incidence of paraanastomotic aneurysms to be $15 \%$ after a mean follow-up of 7 years after surgery for rAAA [24].

The aneurysm-related mortality later than 30 days postoperatively was $7 \%$, mainly due to deaths between 30 and 90 days from complications directly related to the surgery in a prolonged post-operative period. If excluding the four patients who died in the prolonged post-operative setting, very few patients died from aneurysm-related causes. Others have reported findings similar to ours; vascular- and graft-related mortality was higher in rAAA $(3 \%)$ then after elective repair $(1 \%)$ after a mean follow-up of 7 years [24]. How one defines aneurysm-related mortality is poorly defined in the literature [24, 34], and varying classifications may certainly have an impact on the numbers reported.

Cause of death is well documented in the randomized trials on rAAA [29, 33, 35], but rarely reported in other investigations on long-term survival in rAAA $[8,22,25]$. The IMPROVE trial [29] reported an aneurysm-related cause of death in $4.4 \%$, cardiovascular $10.9 \%$, pulmonary $8.2 \%$ and cancer $7.1 \%$. A previous population-based study found ischaemic heart disease $(36 \%)$ to be the primary cause of death in both elective and ruptured aneurysm repairs [19]. A German study reported that patients surviving the first 6 months died of the same causes as the age- and gender-matched population [36]. In the present study, none of the deaths registered after 6 months can be directly related to the prior surgical treatment of rAAA. The cause of death was known in about two-thirds of patients who died during follow-up. According to the Norwegian Institute of Public Health [37] cardiovascular, cancer and pulmonary diseases are the cause of death in 25 , 25 and $10 \%$ of cases in Norway, respectively. Thus, deaths from cancer may have been overrepresented in the study group. 
A high rate of cardiovascular comorbidities did not seem to affect survival, nor did other comorbidities. Others have found coronary heart disease [20, 24], but also lung disease and renal failure to be a significant factor in predicting poorer long-time survival after surgical treatment for rAAA [20, 28]. The impact of cardiovascular comorbidities on survival may have been biased by the high perioperative mortality in this study group $(46 \%)$. Those with significant heart disease may have died in the initial perioperative period, thus representing a survival bias.

A Norwegian study from the early 1980s [34] reported that cardiovascular disease was the cause of death in $50 \%$ of all deaths after surgery for rAAA in the perioperative period and later. Of the patients who survived the 30 days post-surgery, $29 \%$ died from heart disease. This is in accordance with our findings, where cardiac disease was the cause of death in $26 \%$ $(N=6)$ of patients with a known cause of death.

The epidemiologically well-defined, unselected population and the long follow-up time of this study are a strength compared to similar studies on outcome after rAAA repair. Few population-based reports have been published with such a long follow-up time; most studies have a mean or median follow-up time of 1-4 years [7, 9].

The major limitation of this study is the limited size of the study population, especially the few women. Only one patient was treated with rEVAR as this procedure was generally not available in the institution during the study period. This may affect the generalizability of our findings.

Long-term survival after surgery for rAAA was good and comparable to the survival of an age- and sex-matched population. A majority of patients surviving rAAA were discharged directly to their home indicating a good functional level.

Acknowledgements Open Access funding provided by University of Bergen.

Open Access This article is licensed under a Creative Commons Attribution 4.0 International License, which permits use, sharing, adaptation, distribution and reproduction in any medium or format, as long as you give appropriate credit to the original author(s) and the source, provide a link to the Creative Commons licence, and indicate if changes were made. The images or other third party material in this article are included in the article's Creative Commons licence, unless indicated otherwise in a credit line to the material. If material is not included in the article's Creative Commons licence and your intended use is not permitted by statutory regulation or exceeds the permitted use, you will need to obtain permission directly from the copyright holder. To view a copy of this licence, visit http://creativecommons. org/licenses/by/4.0/.

\section{References}

1. Reite A, Soreide K, Ellingsen CL et al (2015) Epidemiology of ruptured abdominal aortic aneurysms in a well-defined
Norwegian population with trends in incidence, intervention rate, and mortality. J Vasc Surg 61(5):1168-1174

2. Reimerink JJ, van der Laan MJ, Koelemay MJ et al (2013) Systematic review and meta-analysis of population-based mortality from ruptured abdominal aortic aneurysm. Br J Surg 100(11):1405-1413

3. Brattheim BJ, Eikemo TA, Altreuther M et al (2012) Regional disparities in incidence, handling and outcomes of patients with symptomatic and ruptured abdominal aortic aneurysms in Norway. Eur J Vasc Endovasc Surg 44(3):267-272

4. Hultgren R, Zommorodi S, Gambe M et al (2016) A majority of admitted patients with ruptured abdominal aortic aneurysm undergo and survive corrective treatment: a population-based retrospective cohort study. World J Surg 40(12):3080-3087. https://doi.org/10.1007/s00268-016-3705-9

5. Bown MJ, Sutton AJ, Bell PR et al (2002) A meta-analysis of 50 years of ruptured abdominal aortic aneurysm repair. Br J Surg 89(6):714-730

6. Badger S, Forster R, Blair PH et al (2017) Endovascular treatment for ruptured abdominal aortic aneurysm. Cochrane Database Syst Rev 5:CD005261

7. Karthikesalingam A, Bahia SS, Patterson BO et al (2013) The shortfall in long-term survival of patients with repaired thoracic or abdominal aortic aneurysms: retrospective case-control analysis of hospital episode statistics. Eur J Vasc Endovasc Surg 46(5):533-541

8. Englund R, Katib N (2016) Long-term survival following open repair of ruptured abdominal aortic aneurysm. ANZ J Surg 87(5):390-393

9. Sonesson B, Bjorses K, Dias N et al (2017) Outcome after ruptured AAA repair in octo- and nonagenarians in Sweden 1994-2014. Eur J Vasc Endovasc Surg 53(5):656-662

10. Reite A, Soreide K, Vetrhus M (2017) Comparing the accuracy of four prognostic scoring systems in patients operated on for ruptured abdominal aortic aneurysms. J Vasc Surg 65(3):609-615

11. Vandenbroucke JP, von Elm E, Altman DG et al (2007) Strengthening the reporting of observational studies in epidemiology (STROBE): explanation and elaboration. Epidemiology 18(6):805-835

12. Owens WD, Felts JA, Spitznagel EL Jr (1978) ASA physical status classifications: a study of consistency of ratings. Anesthesiology 49(4):239-243

13. Howard DP, Banerjee A, Fairhead JF et al (2015) Age-specific incidence, risk factors and outcome of acute abdominal aortic aneurysms in a defined population. Br J Surg 102(8):907-915

14. Team RC (2018) A language and environment for statistical computing. R Foundation for Statistical Computing, Vienna, Austria. https://www.R-project.org/

15. Pohar M, Stare J (2006) Relative survival analysis in R. Comput Methods Programs Biomed 81(3):272-278

16. Norway Statistics. Population 2019. https://www.ssb.no/en/ befolkning/nokkeltall/population

17. Anjum A, von Allmen R, Greenhalgh R et al (2012) Explaining the decrease in mortality from abdominal aortic aneurysm rupture. Br J Surg 99(5):637-645

18. Bjorck M (2014) Surgery for ruptured abdominal aortic aneurysm. BMJ 348:g95

19. Norman PE, Semmens JB, Lawrence-Brown MM et al (1998) Long term relative survival after surgery for abdominal aortic aneurysm in western Australia: population based study. BMJ 317(7162):852-856

20. Johnston KW (1994) Ruptured abdominal aortic aneurysm: sixyear follow-up results of a multicenter prospective study. Canadian Society for Vascular Surgery Aneurysm Study Group. J Vasc Surg 19(5):888-900 
21. Mani K, Bjorck M, Lundkvist J et al (2009) Improved long-term survival after abdominal aortic aneurysm repair. Circulation 120(3):201-211

22. Hinterseher I, Kuffner H, Koch R et al (2012) Comparison of survival rates for abdominal aortic aneurysm treatment methods. World J Surg 36(4):917-922. https://doi.org/10.1007/s00268012-1477-4

23. Raats JW, Flu HC, Ho GH et al (2014) Long-term outcome of ruptured abdominal aortic aneurysm: impact of treatment and age. Clin Interv Aging 9:1721-1732

24. Cho JS, Gloviczki P, Martelli E et al (1998) Long-term survival and late complications after repair of ruptured abdominal aortic aneurysms. J Vasc Surg 27(5):813-819 (Discussion 9-20)

25. Dueck AD, Kucey DS, Johnston KW et al (2004) Long-term survival and temporal trends in patient and surgeon factors after elective and ruptured abdominal aortic aneurysm surgery. J Vasc Surg 39(6):1261-1267

26. Egorova NN, Vouyouka AG, McKinsey JF et al (2011) Effect of gender on long-term survival after abdominal aortic aneurysm repair based on results from the Medicare national database. J Vasc Surg 54(1):1-12 e6 (Discussion 1-2)

27. Gwon JG, Kwon TW, Cho YP et al (2016) Analysis of in hospital mortality and long-term survival excluding in hospital mortality after open surgical repair of ruptured abdominal aortic aneurysm. Ann Surg Treat Res 91(6):303-308

28. Law Y, Chan YC, Cheng SW (2017) Predictors of early operative mortality and long-term survival in octogenarians undergoing open and endovascular repair of abdominal aortic aneurysm. Asian J Surg 41(5):490-497

29. Investigators IT (2017) Comparative clinical effectiveness and cost effectiveness of endovascular strategy $\mathrm{v}$ open repair for ruptured abdominal aortic aneurysm: 3 year results of the IMPROVE randomised trial. BMJ 359:j4859

30. Lo RC, Schermerhorn ML (2016) Abdominal aortic aneurysms in women. J Vasc Surg 63(3):839-844

31. Deery SE, Soden PA, Zettervall SL et al (2017) Sex differences in mortality and morbidity following repair of intact abdominal aortic aneurysms. J Vasc Surg 65(4):1006-1013

32. Sweeting MJ, Ulug P, Powell JT et al (2015) Ruptured aneurysm trials: the importance of longer-term outcomes and meta-analysis for 1-year mortality. Eur J Vasc Endovasc Surg 50(3):297-302

33. Desgranges P, Kobeiter H, Katsahian S et al (2015) Editor's choice-ECAR (Endovasculaire ou Chirurgie dans les Anevrysmes aorto-iliaques Rompus): a French randomized controlled trial of endovascular versus open surgical repair of ruptured aorto-iliac aneurysms. Eur J Vasc Endovasc Surg 50(3):303-310

34. Soreide O, Grimsgaard C, Myhre HO et al (1982) Time and cause of death for 301 patients operated on for abdominal aortic aneurysms. Age Ageing 11(4):256-260

35. van Beek SC, Vahl A, Wisselink W et al (2015) Midterm reinterventions and survival after endovascular versus open repair for ruptured abdominal aortic aneurysm. Eur J Vasc Endovasc Surg 49(6):661-668

36. Hinterseher I, Saeger HD, Koch R et al (2004) Quality of life and long-term results after ruptured abdominal aortic aneurysm. Eur J Vasc Endovasc Surg 28(3):262-269

37. Health NIoP. Publications list for Cause of Death Registry 2019. https://www.fhi.no/en/hn/health-registries/cause-of-death-reg istry/Publications-Cause-Death-Registry/

Publisher's Note Springer Nature remains neutral with regard to jurisdictional claims in published maps and institutional affiliations. 